\title{
Saccadic Target Selection Deficits after Lateral Intraparietal Area Inactivation in Monkeys
}

\author{
Claire Wardak, ${ }^{1}$ Etienne Olivier, ${ }^{1,2}$ and Jean-René Duhamel ${ }^{1}$ \\ 1/nstitut des Sciences Cognitives, Unité Mixte de Recherche 5015 Centre National de la Recherche Scientifique- \\ Université Claude Bernard Lyon 1, 69675 Bron Cedex, France, and 2Laboratoire de Neurophysiologie, Université \\ Catholique de Louvain, 1200 Bruxelles, Belgium
}

We investigated the contribution of the lateral intraparietal area (LIP) to the selection of saccadic eye movement targets and to saccade execution using muscimol-induced reversible inactivation and compared those effects with inactivation of the adjacent ventral intraparietal area (VIP) and with sham injections of saline into LIP. Three types of tasks were used: saccades to single visual or memorized targets, saccades to synchronous and asynchronous bilateral targets, and visual search of a target among distractors. LIP inactivation failed to produce deficits in the latency or accuracy of saccades to single targets, but it dramatically reduced the frequency of contralateral saccades in the presence of bilateral targets, and it increased search time for a contralateral target during serial visual search. In the latter task, the observed deficits might reflect either an ispilateral bias in saccadic search strategy or an attentional impairment in locating a target among flanking distractors within the contralateral field. No effects were observed on any of these tasks after VIP inactivation. These results suggest that one important contribution of LIP to oculomotor behavior is the selection of targets for saccades in the context of competing visual stimuli.

Key words: saccades; target selection; parietal; monkey; LIP; inactivation; visual salience
Saccadic eye movements constitute the primary means by which we explore our visual environment. Several cerebral structures participate in the selection of eye movement targets and in the execution of saccades. One of these is the lateral intraparietal area (LIP). LIP receives input from several extrastriate visual areas (Andersen et al., 1990; Felleman and Van Essen, 1991; Bullier et al., 1996) and is reciprocally connected with the frontal eye field and the superior colliculus (Lynch et al., 1985; Blatt et al., 1990). Single-cell recording experiments have shown that LIP neurons carry visual signals that are modulated by attention (Colby et al., 1996; Ben Hamed et al., 2002) as well as motor signals in relation to the planning and execution of saccades (Barash et al., 1991a,b; Colby et al., 1996). However, the exact role of LIP in relation to saccadic behavior remains unclear. One hypothesis proposes that LIP controls selective spatial attention and acts as a salience map of the visual field (Gottlieb et al., 1998; Kusunoki et al., 2000). Another hypothesis suggests that LIP is directly involved in the representation of motor plans for saccades (Mazzoni et al., 1996). Although these two proposals do not appear a priori incompatible because attention and eye movements are often coupled, much of the controversy about the role of LIP is centered on the issue of whether neuronal activity in this area corresponds to a general attentional signal uncommitted to a particular motor effector or whether this signal is intrinsically linked to saccade preparation. The results from single-cell recording experiments are equivocal in this respect. On the one hand, the

Received June 3, 2002; revised Sept. 6, 2002; accepted Sept. 6, 2002.

This work was supported by the French Foundation for Medical Research and the Centre National de la Recherche Scientifique. We thank Eric Rouiller for providing support with the histological analysis.

Correspondence should be addressed to Jean-René Duhamel, Institut des Sciences Cognitives, 67 Boulevard Pinel, 69675 Bron Cedex, France. E-mail: jrd@isc.cnrs.fr.

Copyright $@ 2002$ Society for Neuroscience $0270-6474 / 02 / 229877-08 \$ 15.00 / 0$ observation that LIP activity during a motor preparation period is stronger for saccades than for reaching movements (Snyder et al., 1998) would appear to support the latter view. On the other hand, it has been shown that LIP activity during saccade preparation reflects the dynamics of attention as it is deviated from, and then returns to, the saccade goal (Goldberg et al., 2002).

One empirical argument, which has received relatively little attention in this debate, is the effect of lesions. Large parietal cortex resections, which include LIP, cause minor increases in saccade latency and negligible effects on accuracy (Lynch and McLaren, 1989). Li et al. (1999) also found increased latency and slight hypometria of memory-guided saccades after muscimol-induced inactivation of LIP. Such deficits appear relatively minor compared with the near disappearance of memoryguided saccades after frontal eye field (FEF) inactivation (Dias and Segraves, 1999).

Here, we investigated the contribution of LIP to saccadic behavior using several eye movement tasks to distinguish between effects of LIP inactivation on target selection and saccade execution. We observed no significant deficits during visually or memory-guided saccades to single visual targets. However, the monkeys showed a strong ipsilesional saccadic bias when two targets were presented simultaneously or with a small asynchrony. During visual search, LIP inactivation biased ocular exploration ipsilesionally and increased search time for a contralesional target. These results suggest the implication of both attentional and decisional factors in the observed saccadic target selection deficits.

\section{MATERIALS AND METHODS}

Two adult monkeys (Macaca mulatta, monkeys A and M) weighing 5.5 and $6.5 \mathrm{~kg}$ were used in these experiments following procedures approved by the local animal care committee in compliance with the guidelines of European Community on animal care. Each monkey un- 
derwent a single surgical session under propofol anesthesia to prepare for chronic recording of eye movements and extracellular recording within the parietal cortex. The animals were implanted with scleral search coils (Judge et al., 1980) and a head-restraining device. On the basis of stereotaxic coordinates, a craniotomy was made over the right parietal sulcus, and a stainless steel recording chamber was implanted to allow access to LIP with microelectrodes and injection needles.

Throughout the duration of the experiments, the monkeys were seated in a primate chair with their heads restrained, facing a tangent translucent screen $35 \mathrm{~cm}$ away, which spanned $\pm 55^{\circ}$ of the visual field. Behavioral paradigms, visual displays, and storage of both neuronal discharge and eye movements were under the control of a personal computer running a real-time data acquisition system (REX) (Hays et al., 1982). Visual stimuli were back-projected onto the screen by a digital light processing video projector. Eye movements were recorded with the magnetic search coil technique (Primelec), and horizontal and vertical eye positions were digitized at $250 \mathrm{~Hz}$. All data analyses were performed off-line.

Single neuron activity was recorded extracellularly with tungsten microelectrodes (Frederick Haer; 1-2 M $\Omega$ at $1 \mathrm{kHz}$ ), which were lowered through a stainless steel guide tube by means of a hydraulic microdrive (Narishige). Visual and saccade-related neuronal responses were recorded in the lateral bank of the intraparietal sulcus to determine precisely both the location and extent of LIP. The limits of VIP were also identified on the basis of its distinctive neuronal activity in relation to visual motion and somatosensory stimulation (Duhamel et al., 1997, 1998).

Behavioral tasks. In the visually guided saccade paradigm, monkeys were required to maintain central fixation for $1000-1600 \mathrm{msec}$ until the fixation point disappeared. At this time, a visual target appeared at one of eight possible locations $\left(14^{\circ}\right.$ of eccentricity, radially distributed about the fixation point at $45^{\circ}$ intervals) in randomly interleaved order. The monkeys received a liquid reward if they made a saccade toward the target within $1000 \mathrm{msec}$ of its appearance and maintained fixation there for at least $500 \mathrm{msec}$.

The memory-guided saccade task required the monkey to fixate centrally for $300 \mathrm{msec}$. A target was then flashed for $100 \mathrm{msec}$ at one of eight locations $\left(14^{\circ}\right.$ of eccentricity, radially distributed about the fixation point at $45^{\circ}$ intervals). The monkeys maintained fixation for another 1200 $1800 \mathrm{msec}$, until the fixation point disappeared, which was the cue to look at the memorized target. If the monkeys made a saccade at the appropriate time to the location of the target, the target reappeared, and monkeys had to maintain fixation for another $500 \mathrm{msec}$ to obtain a reward. The eye position tolerance windows were set at 2.5 and $5^{\circ}$ at the central and peripheral locations, respectively.

The double-target paradigm was used to test for extinction as observed in human patients with neglect. These patients are unable to report a contralesional event when two bilateral events are in competition. The monkeys fixated centrally for $500-1100 \mathrm{msec}$. On half of the trials, a single eccentric target was flashed $(50 \mathrm{msec}$ for monkey M, $100 \mathrm{msec}$ for monkey A) at one of two possible locations $\left(14^{\circ}\right.$ to the left or right of fixation). On the other half of the trials, both targets were flashed with a variable delay. Asynchrony ranged between $-40 \mathrm{msec}$ (contralesional lag) and $+280 \mathrm{msec}$ (contralesional lead), by steps of $40 \mathrm{msec}$. The monkeys could make a saccade to any one of the two but were rewarded for only $50 \%$ of the trials to avoid reinforcing a possible natural directional bias. Fixation point extinction and peripheral target appearance were offset by a $100 \mathrm{msec}$ gap to prevent the perception of an illusory movement toward the first target that could influence the monkeys' choice.

In the visual search task, a central fixation period of $1000-1600 \mathrm{msec}$ was followed by presentation of a visual array containing one target $\left(0.8^{\circ}\right.$ red square) and several distractors. The stimuli were distributed uniformly around a virtual circle of $14^{\circ}$ radius, such that the angles between adjacent stimuli were 90,45 , and $22.5^{\circ}$ for the $4-, 8$-, and 16 -stimulus arrays, respectively. The search array remained visible for 2-3.5 sec, depending on array size. Monkeys were allowed to perform several saccades and were rewarded when the target was fixated for at least 500 msec. Two standard search conditions were used, differing in their attentional demands. In the conjunction search condition, a third of the distractors had the same shape as the target but a different color (orange squares), a third had the same color but different shape (red triangles), and the remaining third differed in both shape and color (orange triangles). The target was thus defined by a conjunction of form and color. In the feature search condition, a single distractor type (orange triangle) was used. Linear regression was used to characterize the effect of array size on search time. In agreement with previous results in both humans and monkeys (Treisman and Gelade, 1980; Bichot and Schall, 1999), feature search yielded virtually flat regression slopes of 1.08 and -1.04 msec per item, whereas in the conjunction condition search slopes rose to 5.4 and $11.5 \mathrm{msec}$ per item. In one variant of the conjunction task, tested only in monkey M, all items were placed on either the left or right side of the fixation point, along a virtual semicircle of $14^{\circ}$ radius for the 4 and 8 items conditions and of two semicircles of 12 and $16^{\circ}$ radius for the 16 items conditions. The target and distractors sets were constructed from diamond and square shapes that could be yellow or green. We used different stimuli because monkey $M$ had developed over time a rather shallow search slope with the previously used stimuli, which may be an effect of overtraining. The novel objects produced a slope of $17.1 \mathrm{msec}$ per item, which is closer to those typically obtained in human studies.

LIP inactivation. A solution of $2-6 \mu \mathrm{g} / \mu \mathrm{l}$ of muscimol (Sigma) in saline was injected with a $5 \mu \mathrm{l}$ Hamilton syringe connected to a 29 gauge stainless steel needle. Muscimol, a GABA $\mathrm{A}_{\mathrm{A}}$ agonist, was used because it interacts specifically with $\mathrm{GABA}_{\mathrm{A}}$ receptors and does not induce conduction block in fibers of passage. Two to three needle tracks were performed in each experiment, and along each track, two injections were made at distinct physiologically characterized sites of LIP, separated by 2-4 mm. Tracks were made to depths of $\sim 4-8 \mathrm{~mm}$ below the cortex surface; the volume injected at each site was $0.5-1 \mu \mathrm{l}$ and was delivered, by steps of $0.2 \mu \mathrm{l}$, every $2 \mathrm{~min}$. The total amount of muscimol injected in each experiment ranged between 12 and $24 \mu \mathrm{g}$. In both animals, the injections were made into the right parietal cortex. After the injections were completed, double-target saccade trials were run to detect the onset of muscimol effects, because extinction was a reliable on-line behavioral marker of those effects, which generally started 15-60 min after injection. All of the different tasks could not be performed on a given inactivation experiment; however, each one was tested several times (two to five for visually and memory-guided saccade tasks, four to six for extinction tasks, and five to seven for visual search tasks). The order of the different tasks was counterbalanced across inactivation experiments, and control data were always obtained on the following day and under the same conditions. The entire duration of behavioral testing never lasted $>2 \mathrm{hr}$, well within the accepted temporal range of muscimol effects (Malpeli, 1999; Martin and Ghez, 1999). Seven LIP inactivation experiments were conducted in monkey $\mathrm{M}$, and five were conducted in monkey A. A physiological saline injection into LIP and a muscimol inactivation of adjacent VIP in monkeys $\mathrm{A}$ and $\mathrm{M}$, respectively, served as a further control for the specificity of the effects.

Data analysis. Preliminary analysis of the data did not indicate a systematic tendency for LIP inactivation to affect particular target locations within the contralesional hemifield. Thus, for the sake of presentation clarity, data for different target locations were grouped by hemifield. Statistical comparisons between performance on control and inactivation sessions were achieved by means of two-way, inactivation condition $\times$ hemifield, ANOVAS, unless specified otherwise. Because the monkeys performed many different tasks on a given inactivation session, relatively few trials were obtained for each level of the different factors manipulated in any given task. This was particularly true of the visual search task in which target location, number of distractors, and search condition were systematically varied. Therefore, data from inactivation sessions and for following-day control sessions were pooled together to increase statistical power.

Histological procedure. In monkey A, an anatomical study was performed at the end of the experiments to verify the approximate location of muscimol injection sites in LIP (Fig. 1). Fluorescent tracer (Fast Blue, Sigma; $5 \%$ in saline) was injected in two tracks, which had served previously to inactivate LIP. The two tracks were separated by $\sim 6 \mathrm{~mm}$, and in each track, two injections of $0.2 \mu \mathrm{l}$ were performed at two depths, separated by $1.5 \mathrm{~mm}$. After a $24 \mathrm{hr}$ survival period, anesthesia was induced by ketamine injection $(10 \mathrm{mg} / \mathrm{kg}, \mathrm{i} . \mathrm{m}$.), and then the animal was administered a lethal dose $(60 \mathrm{mg} / \mathrm{kg}$, i.p.) of sodium pentobarbital. Four electrodes were lowered in the recording chamber, at each corner of the grid. Once a deep anesthesia was attained, the animal was perfused through the heart with a vascular rinse $(0.9 \% \mathrm{NaCl}$ in $0.1 \mathrm{M}$ phosphate buffer, $5000 \mathrm{U}$ of heparin at $36^{\circ} \mathrm{C}$ ). This was followed by fixative (3 1 of $4 \%$ paraformaldehyde in $0.1 \mathrm{M}$ phosphate buffer and $10 \%$ sucrose in 21 of $4 \%$ paraformaldehyde in $0.1 \mathrm{~m}$ phosphate buffer, $\mathrm{pH} 7.3$, at $4^{\circ} \mathrm{C}$ ). After $45 \mathrm{~min}$, the perfusion with fixative was stopped and continued with $0.1 \mathrm{M}$ phosphate buffer (at $4^{\circ} \mathrm{C}$ ) containing $20 \%$ sucrose. After perfusion, the head was fixed in a stereotaxic apparatus, and the dorsal part of the cranium was removed. One block containing the portion of the intraparietal cortex delimited by the electrodes was made in the stereotaxic 


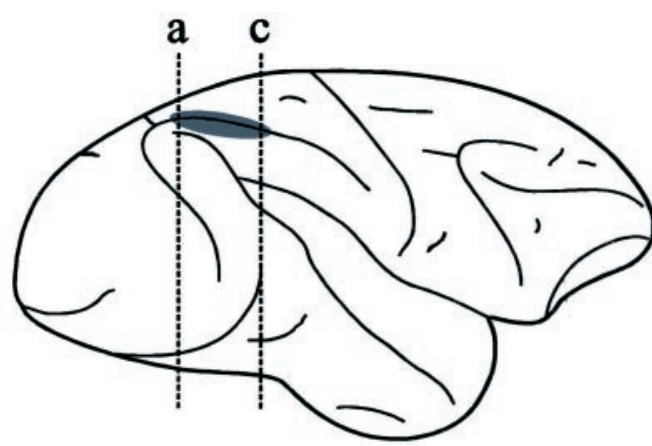

a

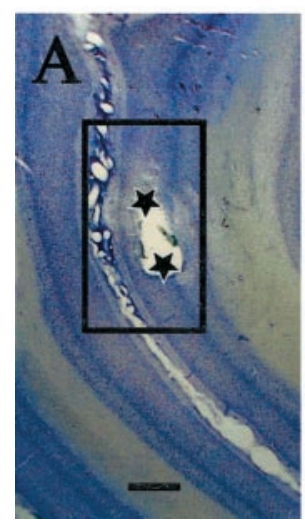

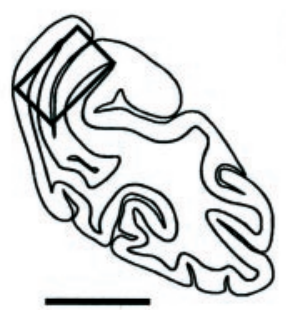

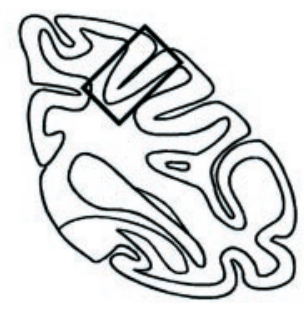

C

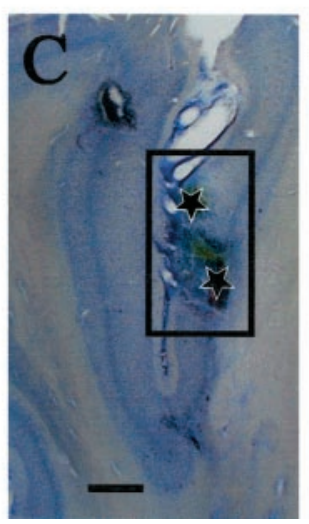

Figure 1. Estimate of injection site location in LIP. The top left panel shows a lateral view of the right hemisphere in monkey A. The gray-shaded area highlights the approximate position of LIP as characterized electrophysiologically and projected onto the brain surface. Vertical dashed lines indicate the level of the coronal sections through the injection sites of Fast Blue. These injections of fluorescent tracer reproduced the location of the two more distant muscimol injections performed in this animal. The top right panel shows the coronal sections indicated by the vertical dashed lines on the lateral view of the brain. The boxed outlines on the coronal sections $a$ and $c$ indicate, respectively, the location of the photomicrographs $A$ and $C$ shown in the bottom panel. Scale bar, $10 \mathrm{~mm}$. The bottom panels illustrate photomicrographs of injection sites at two different magnification factors. $A$, $C$, Low-power photomicrographs showing coronal sections from posterior and middle portions of the intraparietal sulcus stained with the Nissl method. Black stars identify the approximate location of the two Fast Blue injections performed along each track. The boxed outlines indicate the location of the photomicrographs $B$ and $D$. $B, D$, Higher-power photomicrographs showing the core of the fluorescent tracer injections. Scale bars, $1 \mathrm{~mm}$. coronal plane (approximately P15-P25); the block was kept in 30\% sucrose in $0.1 \mathrm{M}$ phosphate buffer at $4^{\circ} \mathrm{C}$ until sinking. Coronal frozen sections were cut at $80 \mu \mathrm{m}$, stored refrigerated in $0.1 \mathrm{~m}$ phosphate buffer, and then mounted onto glass slides coated with gelatin-chrome alum. High-power micrographs of sections containing injection sites were obtained from a fluorescent microscope and a macrophotography system by Olympus. Selected sections were then stained with cresyl violet, and lowerpower micrographs were taken to determine the injection site location.

\section{RESULTS}

\section{No effects on saccades to single targets}

LIP inactivation did not alter the latency of visually and memoryguided saccades directed to one of the eight possible targets (two-way ANOVA; no significant main effect of inactivation or hemifield $\times$ inactivation interaction in monkeys $\mathrm{A}$ and $\mathrm{M}$, for both eye movement tasks). Figure $2 A$ shows the mean latency of visually guided saccades across three inactivation experiments for monkey $\mathrm{M}$ and two inactivation experiments for monkey $\mathrm{A}$. Figure $2 B$ presents mean latency of memory-guided saccades obtained in two inactivation experiments for each monkey. Other saccade parameters, i.e., accuracy, duration, amplitude, and omission rate, remained unchanged after LIP inactivation (two-way ANOVA; no significant main effect of inactivation or hemifield $\times$ inactivation interaction in monkeys A and M) (Fig. 3). Similar results were obtained when statistical analyses were performed on individual targets rather than on data grouped by hemifield. The same analyses conducted on individual inactivation experiments did not reveal any deficits. Neither muscimol injections in VIP nor saline injections in LIP produced deficits in visually and memory-guided saccades (two-way ANOVA; no significant inactivation main effect nor hemifield $\times$ inactivation interaction).

\section{Ipsilesional bias on saccades in the presence of two targets}

The behavioral effects of LIP inactivation were also investigated in a saccadic choice task in which monkeys were shown two briefly

\section{A Visually-guided saccade latency}
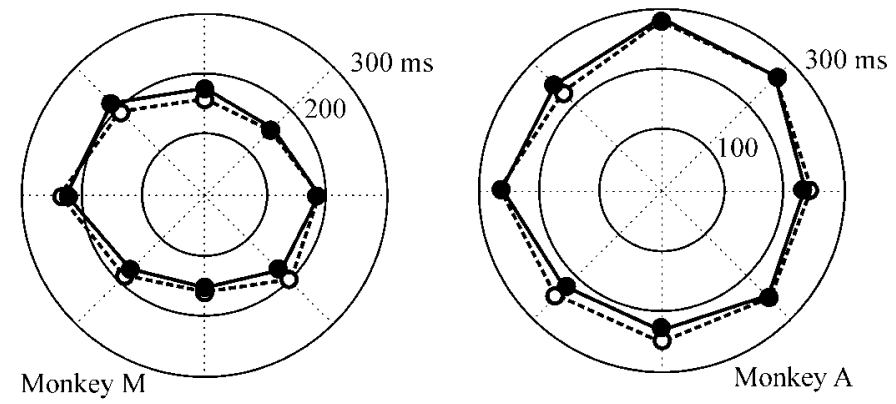

B

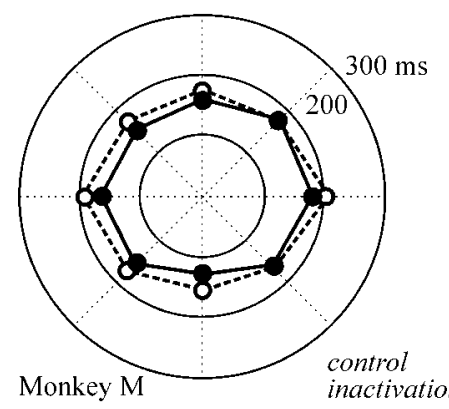
Memory-guided saccade latency

Figure 2. Saccadic eye movement latency for eight target directions spaced $45^{\circ}$ apart. On all panels, the contralesional field is on the left: visually guided saccade latencies $(A)$ and memory-guided saccade latencies $(B)$ for monkeys $\mathrm{M}$ and A, displayed in polar coordinates. Dashed lines represent control data; solid lines represent data obtained during LIP inactivation. 


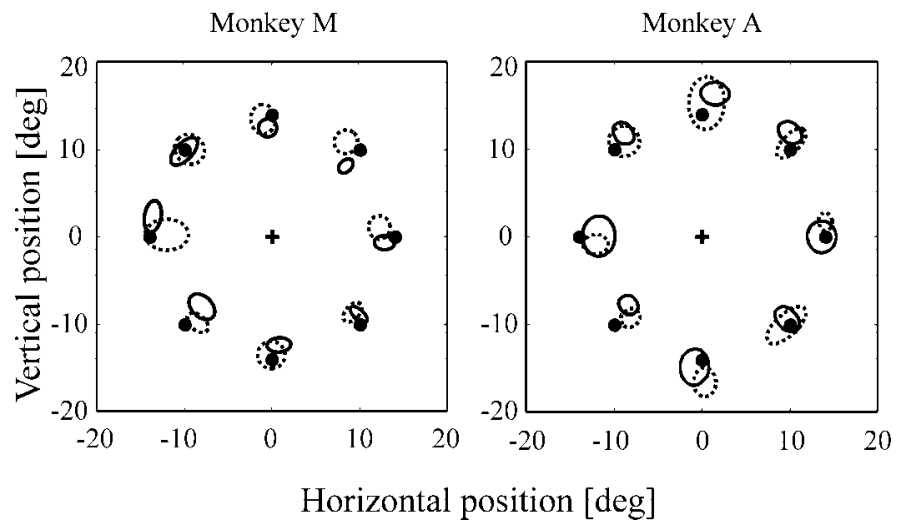

Figure 3. Memory-guided saccade accuracy. The contralesional field is on the left. Ellipses represent mean $\pm 1 \mathrm{SD}$ of saccadic endpoints. The central cross corresponds to fixation point, and black dots correspond to target location. Dashed lines represent control data; solid lines represent data obtained during LIP inactivation. deg, Degree.

flashed targets at $14^{\circ}$ left and right of the fixation point, either simultaneously or with a variable onset asynchrony. Extinction effects were tested during the same inactivation experiments on which we also tested visually and memory-guided saccade performance, and during additional experiments for a total of six inactivations in monkey $\mathrm{M}$ and four inactivations in monkey $\mathrm{A}$. The effects were highly reproducible because significant deficits were observed on every occasion.

The results pooled across all inactivation experiments are illustrated in Figure 4. The probability of eliciting either a left or a rightward saccade depended on the delay between the onset of the two targets. Although the transition between predominantly left and predominantly right choices was more abrupt in monkey $\mathrm{M}$ than in monkey A (Fig. 4), in both animals the relationship between target asynchrony and saccade probability shows a sigmoidal pattern. The point of inflection of the logistic regression curve fitted to the data was used as an indicator of the target onset asynchrony at which a switch in saccadic preference occurred. In the control condition, the two monkeys showed a natural bias toward the right side, because the left target had to precede the right target slightly to obtain an equal saccade probability in both directions (target asynchrony: +50 and $+25 \mathrm{msec}$ in monkeys $\mathrm{M}$ and $\mathrm{A}$, respectively).

Disruption of LIP activity by muscimol injections led to a dramatic decrease in the number of saccades made toward the left, contralesional target when two targets were presented. Indeed, in both monkeys, LIP inactivation severely altered the shape of the relationship between target asynchrony and saccade probability $\left(\chi^{2} ; p<0.01\right)$. In monkey $\mathrm{A}$, an equal saccade probability was obtained for a delay of $\sim 200 \mathrm{msec}$, i.e., $180 \mathrm{msec}$ later than in the control condition, and the sigmoidal function showed a plateau at $\sim 60 \%$. In monkey $\mathrm{M}$, even when the contralesional target preceded the ipsilesional target by $280 \mathrm{msec}$, which was the longer delay investigated, the probability of eliciting a saccade toward the contralesional target remained as low as $20 \%$ (Fig. 4). Given these low maximal proportions of contraversive saccades, the inflection point of the sigmoid curve does not correspond to an equal probability of saccades in either direction. It merely indicates the target onset asynchrony value falling halfway between the two extremes of the contraversive saccade probabilities under muscimol inactivation. Relative to control inflection points,
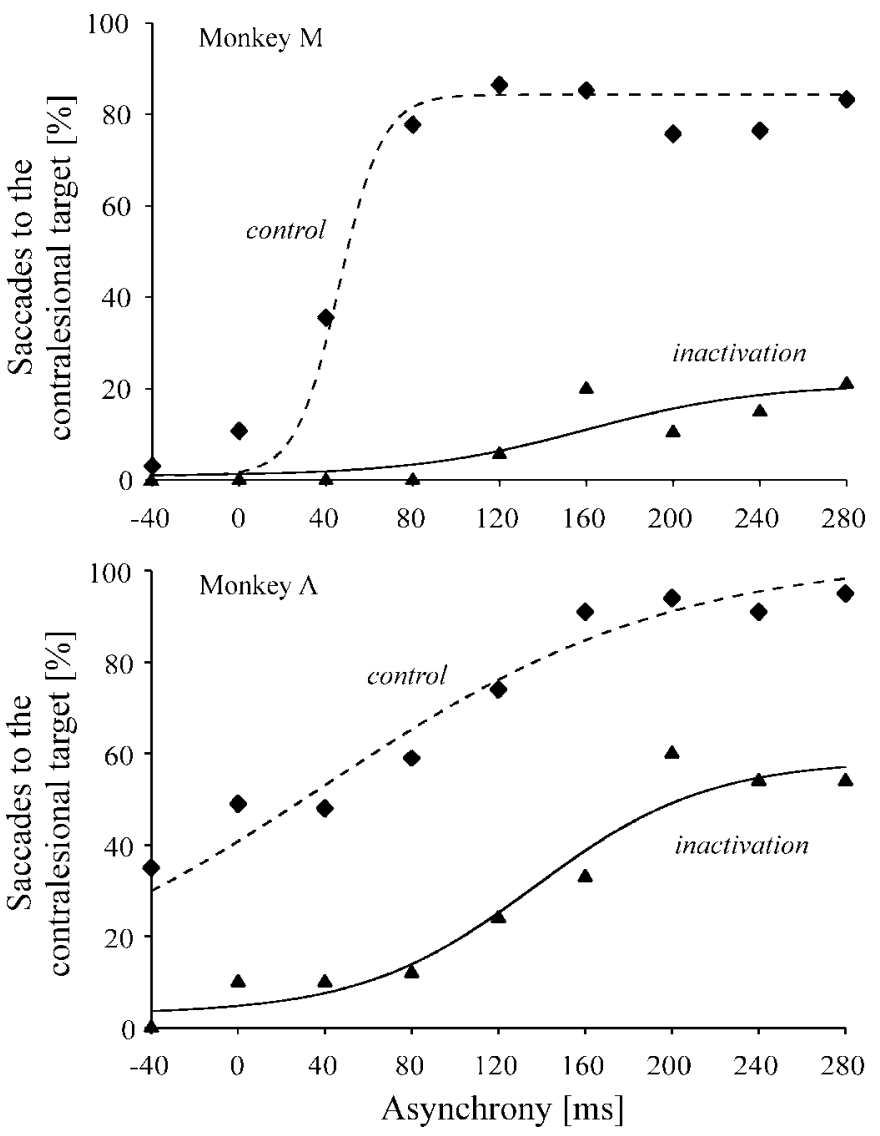

Figure 4. Saccades to bilateral targets. Proportion of contraversive saccades on double-target presentations in monkeys $\mathrm{M}$ and $\mathrm{A}$ for the onset asynchrony of each target, and corresponding logistic regression fits through inactivation (solid line: $p<0.0001, r^{2}=0.98$ for monkey $\mathrm{M} ; p<$ $0.001, r^{2}=0.94$ for monkey A) and control (dashed line: $p<0.001, r^{2}=$ 0.88 for monkey $\mathrm{M} ; p<0.0001, r^{2}=0.95$ for monkey A) data. Positive asynchrony means contralesional target leading the ipsilesional one.

this change in behavior occurred 120 msec later in monkey A and $176 \mathrm{msec}$ later in monkey $\mathrm{M}$.

LIP inactivation also reduced the number of saccades successfully made toward the contralesional target on interleaved trials in which a single target was presented, whereas it did not have such effects on visually guided saccades toward targets that are always presented singly (see above). In monkey $\mathrm{M}$, when a single contralesional target was flashed, the percentage of error trials increased from $23.1 \%$ in the control condition to $82.6 \%$ after LIP inactivation $\left(\chi^{2} ; p<0.0001\right)$; in monkey $\mathrm{A}$, it increased from 4.4 to $27.5 \%\left(\chi^{2} ; p<0.0001\right)$ (Fig. 5). On such trials, the monkeys either did not generate an eye movement or released an inappropriate saccade. These saccades (93\% of the error trials) had a long latency $(>300 \mathrm{msec})$ and did not have the stereotyped characteristics of goal-directed saccades. Rather, such saccades tended to have erratic velocity profiles and curved trajectories, similar to the saccades that monkeys typically produce at the end of trials when breaking fixation. A small minority of saccades (3\% of the error trials) had a short latency and were directed to the mirror position of the target, but this was also true of error trials from control sessions. Finally, it is worth noting that in monkey A, a significant decrease in the proportion of incorrect trials was also observed after LIP inactivation when a single ipsilesional target was presented ( 6.8 vs $1.4 \%$ after LIP inactivation; $\chi^{2} ; p<0.05$ ) 


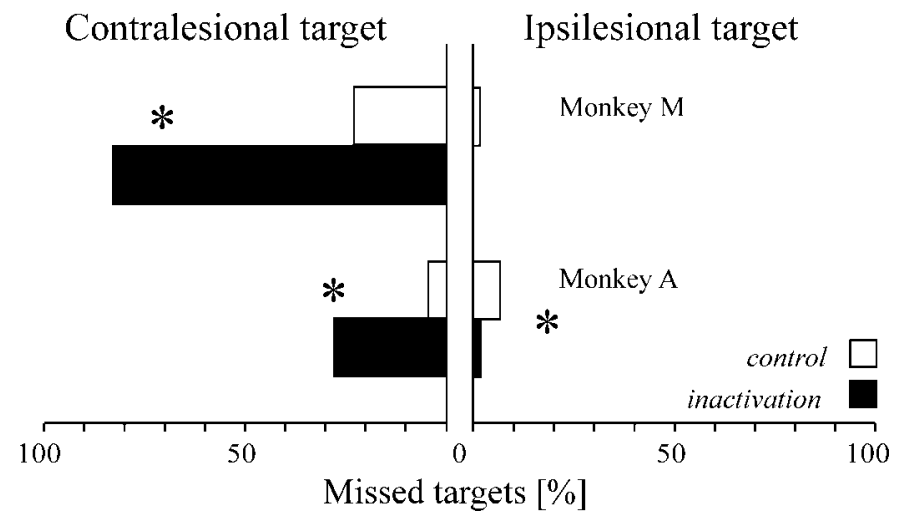

Figure 5. Omissions on single-target trials intermingled with doubletarget presentations. Horizontal bars show the percentage of single-target trials on which the monkeys failed to respond. White bars correspond to control data; black bars correspond to data after LIP inactivation. $* p<0.05$.

(Fig. 5). These results indicate that the status of a single flashed target in the context of a double-target task is different from that of a similar target in the context of a single-target task such as visually or memory-guided saccades, where the percentage of error trials on contralesional side is unaffected by LIP inactivation (Table 1).

Results obtained after muscimol injections in VIP or saline injections in LIP were indistinguishable from those on no injection control experiments $\left(\chi^{2} ; p>0.05\right)$.

\section{Target selection impairment during visual search}

In the visual search task, the monkeys, by means of saccades, had to look for a target presented among several nontarget distractors. Two search conditions were investigated: in the feature search, the distractors shared no feature with the target, although in the conjunction search, the distractors shared either its color or its shape, or neither of them.

Performance in the feature search task showed no contralesional effect of LIP inactivation in either monkey on individual inactivation experiments or on pooled data (target location $\times$ inactivation interaction; $p>0.05$ ). This task was performed during four separate inactivation experiments in monkey $\mathrm{M}$ and two experiments in monkey A. The overall search time was reduced in monkey $\mathrm{M}$ $\left(171.0\right.$ vs $188.2 \mathrm{msec}$ in control; inactivation main effect: $F_{(1,864)}=$ $11.8 ; p<0.001)$ and nonsignificantly increased in monkey A (240.6 vs $223.4 \mathrm{msec}$ in control; inactivation main effect: $p>0.05)$. This was a rather easy task, with a single saccade sufficient to acquire the target on $85-95 \%$ of the trials.

In contrast, search time was systematically increased in the conjunction condition. On pooled data analysis, we found that for monkey M, the overall mean search time was $288.3 \mathrm{msec}$ after inactivation as compared with $226.8 \mathrm{msec}$ in control. For monkey A, search time rose from $361.5 \mathrm{msec}$ to $534.5 \mathrm{msec}$. In both monkeys, search time increased significantly as a function of the number of items, independent of the inactivation condition (monkey M: $F_{(2,902)}=34.9, p<0.01$; monkey A: $F_{(2,678)}=17, p<$ $0.0001)$. More importantly, the effects of muscimol were highly specific to the target location. In monkey $\mathrm{M}$, there was a selective effect of inactivation for contralesional targets $\left(F_{(1,902)}=10.7 ; p<\right.$ $0.001)$ and a significant inactivation $\times$ number of items interaction $\left(F_{(2,902)}=7.9 ; p<0.001\right)$. Post hoc pairwise comparisons (Student-Newman-Keuls; $p<0.05$ corrected) showed that the increase in search time was significant for 8 and 16 items, but not 4 items (Fig. 6). For ipsilesional targets, no significant inactivation or inactivation $\times$ number of items interaction effects were found. In monkey A, search time increased after LIP inactivation for contralesional targets (inactivation: $F_{(1,678)}=141 ; p=0.0002$ ), regardless of the number of items in the display (no inactivation $\times$ number of items interaction). No significant effect of inactivation was observed on performance for ipsilesional targets. These results were obtained during five and four inactivation experiments in monkey $\mathrm{M}$ and monkey A, respectively. Effects observed on individual inactivation experiments mirrored the above pooled results, despite the fact that the data samples were relatively small because of the large number of conditions. In monkey $\mathrm{M}$, significantly longer contralesional search time was observed for 16 items on all inactivation experiments and in three of five experiments for 8 items. In monkey A, the contralesional increase in search time failed to reach significance in only one of the four experiments.

Because the number of saccades allowed to acquire the target was unconstrained, we could examine the oculomotor search strategy adopted by the monkeys in terms of where the eyes landed before arriving on the target. Most of the errant saccades were directed toward ipsilesional distractors. This is illustrated in Figure 7 with single trial examples from monkey $M$, in which the initial saccades were both right-sided regardless of target location. This monkey showed a contralesional side bias in control experiments that was reversed after LIP inactivation, with intermediate saccades now landing more often ipsilesionally (Fig. 8A) $(p<$ $0.0001 ; \chi^{2}$ ). In monkey $\mathrm{A}$, a pronounced ipsilesional bias was already present during control experiments, and LIP inactivation did not exacerbate this tendency. Inspection of the type of distractors visited indicates that saccades landed preferentially on the ones having either the same shape or the same color as the target, with monkey A in particular showing a natural preference for same color over same shape distractors (Fig. $8 B$ ). There was no significant influence of LIP inactivation on the distribution of saccades among the various types of distractors in either monkey.

The drawback of allowing multiple saccades is that it is not possible to determine the extent to which longer visual search time reflects inefficient processing of contralesional stimuli or a bias to make eye movements toward the ipsilesional field. The contribution of the latter factor is less of an issue on trials during which monkeys started searching directly within the hemifield containing the target. A separate analysis conducted on these trials showed only that search time for contralesional targets is still significantly prolonged in both monkeys (351.4 vs $276.1 \mathrm{msec}$ for monkey A, $p<0.001 ; 234.8$ vs $217.4 \mathrm{msec}$ in control for monkey $\mathrm{M}, p<0.05)$. A supplementary task was designed for monkey $\mathrm{M}$, whose search strategy showed a strong ipsilesional bias; in this task the entire conjunction search display was confined to a single visual hemifield. The task was performed by the monkey on two separate inactivation experiments. The pooled results shown in Figure 9 indicate that LIP inactivation increased search time for the target embedded in a contralesional array (inactivation main effect: $F_{(1,857)}=9.75 ; p=0.0019$; no inactivation $\times$ number of items interaction) but had no effect on performance on the ipsilesional array. The same pattern of results was obtained on each inactivation experiment when analyzed individually. It is worth noting that during this task, the monkey never made saccades to the empty hemifield, whether ipsilesional or contralesional; eye movements were restricted to the regions of space containing objects.

The effects of muscimol inactivation in VIP and sham injections of saline in LIP were tested on the conjunction search task 
Table 1. Percentage of correct trials in different tasks for monkey $\mathrm{A}$ and monkey $\mathrm{M}$

\begin{tabular}{|c|c|c|c|c|c|c|c|c|}
\hline & \multicolumn{4}{|c|}{ Monkey A } & \multicolumn{4}{|c|}{ Monkey M } \\
\hline & \multicolumn{2}{|c|}{ Contralesional } & \multicolumn{2}{|c|}{ Ipsilesional } & \multicolumn{2}{|c|}{ Contralesional } & \multicolumn{2}{|c|}{ Ipsilesional } \\
\hline & Control & Inactivation & Control & Inactivation & Control & Inactivation & Control & Inactivation \\
\hline Visually guided saccades & 97.7 & 96.8 & 96.6 & 95.7 & 95.8 & 96.4 & 98.9 & 98.6 \\
\hline Memory-guided saccades & 74.7 & 63.8 & $74.2^{*}$ & $52.1^{*}$ & 63.9 & 67.0 & 57.8 & 55.6 \\
\hline Visual search & 96.3 & 93.3 & 93.9 & 97.0 & 96.1 & 97.8 & 97.5 & 98.0 \\
\hline Single-target presentations & $95.6^{*}$ & $72.5^{*}$ & $93.2^{*}$ & $98.6^{*}$ & $76.9^{*}$ & $17.4^{*}$ & 98.7 & 100 \\
\hline
\end{tabular}

Only trials in which monkey fixated the central target until the appearance of the peripheral stimulus (or stimuli) are considered. ${ }^{*} p<0.05$.

A

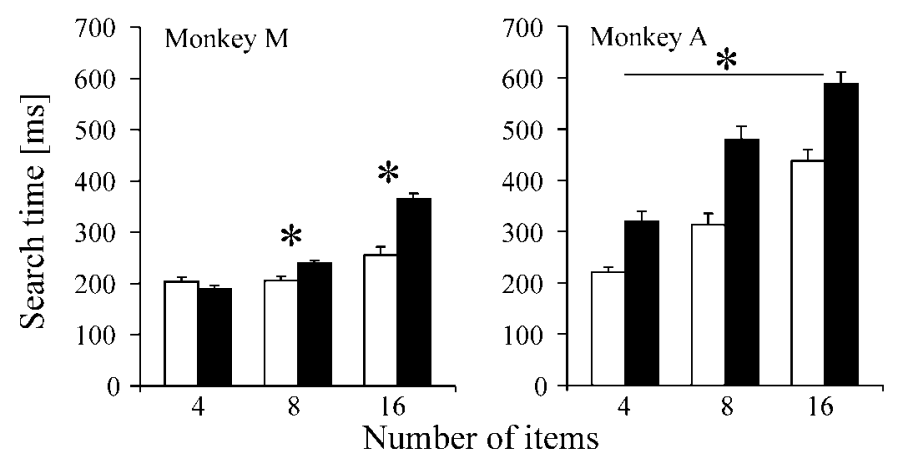

B

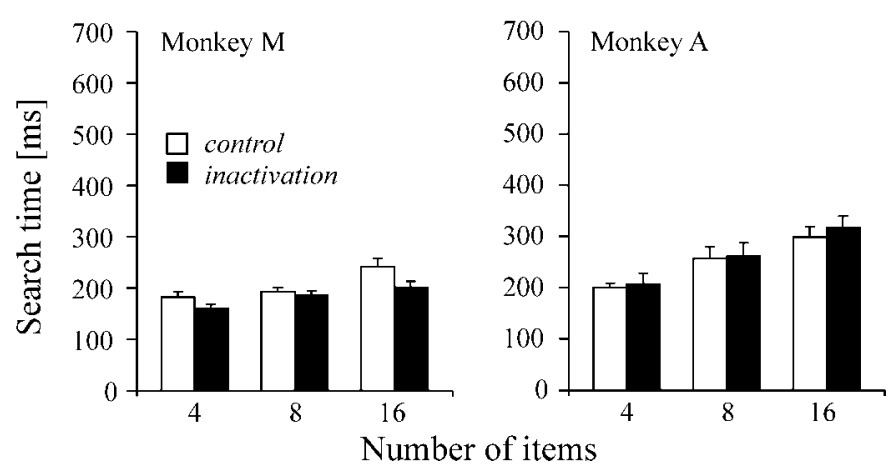

Figure 6. Visual search performance. Search time is shown as a function of display size for contralesional $(A)$ and ipsilesional $(B)$ targets. Search time for a target defined by a conjunction of color (red vs orange) and form (circle vs square) is defined as the interval between the search pattern onset and the start of the saccade landing on the target (bar height is mean search time; error bar indicates SE). White bars correspond to control data; black bars correspond to data after LIP inactivation. Asterisk indicates corrected $p<0.05$.

with a circular array, and performance did not differ from that of the no-injection control experiments.

\section{DISCUSSION}

The main result of the present study is that muscimol-induced inactivation of LIP impairs saccade target selection, whereas saccade programming and execution per se remain unaffected. The absence of oculomotor impairments may seem surprising because LIP has been described as a "parietal eye field" (Andersen et al., 1992) and because a previous inactivation study showed latency and accuracy deficits on memory-guided saccades (Li et al., 1999). Different interpretations can be proposed to resolve this apparent contradiction, in terms of differences in either the degree or topog-
Contralesional target

Ipsilesional target

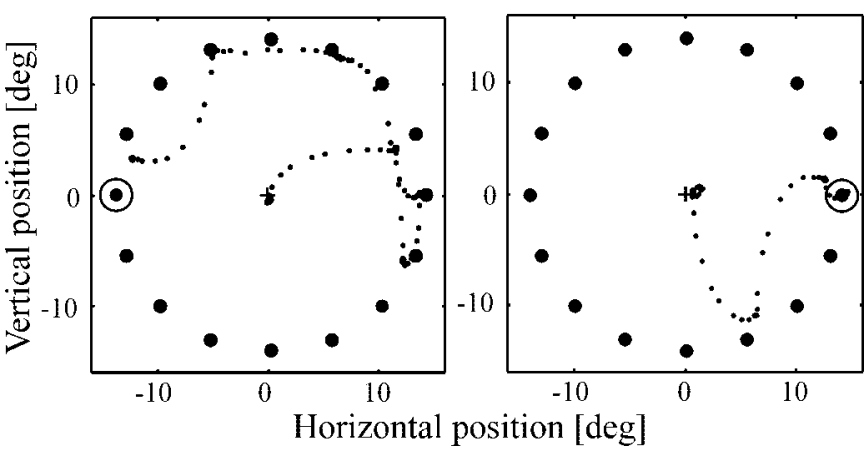

Figure 7. Single-trial examples of visual search patterns from monkey M during LIP inactivation. The small dots represent eye position sampled every $4 \mathrm{msec}$; large dots represent the search stimuli, and the circle represents the location of the target. The contralesional field is on the left.

raphy of inactivation. The first explanation suggests that a common neural substrate exists in LIP for saccade programming and target selection and that selection can be impaired even with a moderate level of inactivation, whereas saccadic deficits only emerge at a higher level of inactivation. The second interpretation implies segregated subsystems within LIP for these two functions. Neither possibility can be excluded given the differences in injection protocols used in the two studies. Li et al. (1999) made principally a unique injection at a high concentration of muscimol, whereas we made multiple smaller injections at a lower concentration. Because the effects observed by these authors were admittedly subtle, slight variations in behavioral protocols used to assess memory-guided saccade performance might also account for some of the discordant findings. Li et al. (1999) tested their monkeys in total darkness, whereas in our study a moderate level of background illumination was always maintained by the video projection system. The absence of a visual reference frame in total darkness, which requires a greater reliance on extra-retinal (eye position) cues, might provide a more sensitive test of saccadic performance. Although it is not possible at this stage to decide between these different explanations, it is an interesting observation on its own that target selection deficits can be observed in the absence of any changes in saccade accuracy and onset latency. Importantly, the effect of LIP inactivation on saccade performance does not follow a simple correlation with overall task difficulty, as assessed by the percentage of error trials (Table 1).

\section{Extinction}

After LIP inactivation, when presented with two possible targets, monkeys directed their saccades almost exclusively toward the ipsilesional one. The issue that needs to be addressed, and to 
A

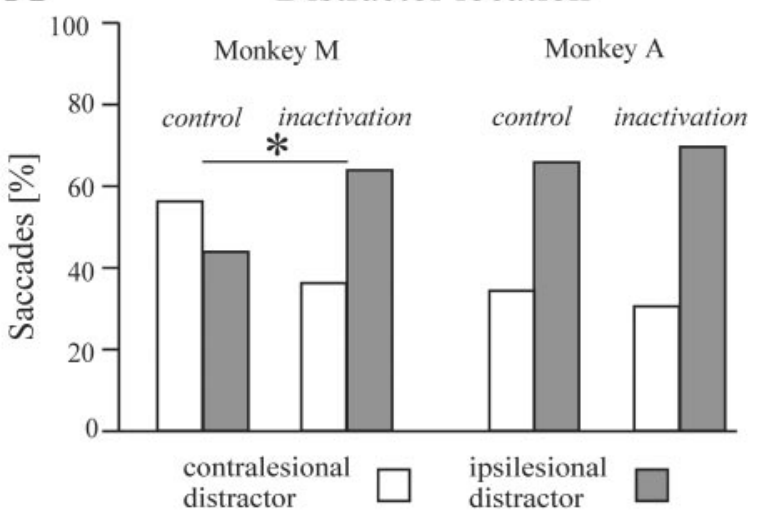

B

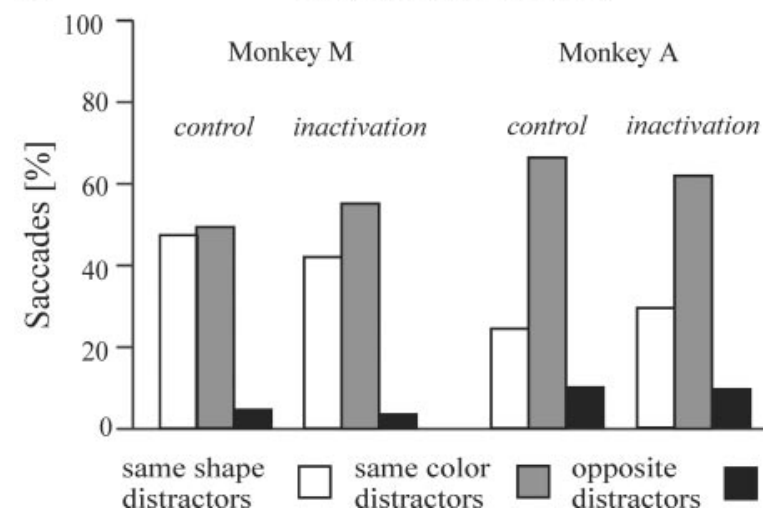

Figure 8. Characteristics of saccades to distractors. A, Proportion of saccades directed to contralesional (white bars) and ipsilesional (gray bars) distractors. $B$, Proportion of saccades directed to same-shape (white bars), same-color ( gray bars), and opposite (i.e., different shape and color; black bars) distractors. ${ }^{*} p<0.05$.

which our data do not speak directly, is the origin of this ipsilateral bias. In human subjects, visual extinction is generally assessed through verbal report and interpreted in terms of perceptual awareness (Karnath, 1988; Di Pellegrino and De Renzi, 1995). The task used in the present study measured a preferred motoric choice, and therefore does not allow us to distinguish whether LIP inactivation induced principally a detection deficit or an ipsilateral motor decision bias. The latter interpretation would imply that both targets are detected but that some kind of critical triggering threshold is reached earlier for saccades directed ipsilaterally rather than contralaterally. Intuitively, if signals related to ipsilateral and contralateral saccades were processed independently and the main effect of LIP inactivation would be to dampen the buildup of excitation for contralesional saccades, one would expect abnormally long latencies for contralateral saccades even in the absence of ipsilateral competition. The fact that we did not observe this in either of our monkeys and that deficits emerged only in the context of bilateral targets would suggest rather that left and right saccade signals do not build up independently and there is some kind of competitive interaction between the two sides. Again, the critical site for this competition, attentional or decisional, remains unclear.

Asynchronous targets have been used to study extinction-like effects in humans in tasks involving temporal order judgment or saccadic choice. Rorden et al. (1997) found that in parietal
A

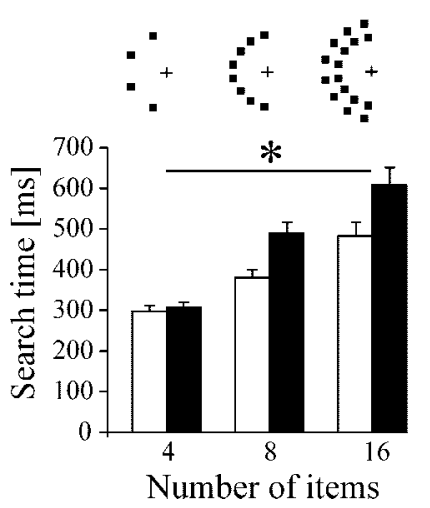

B Ipsilesional
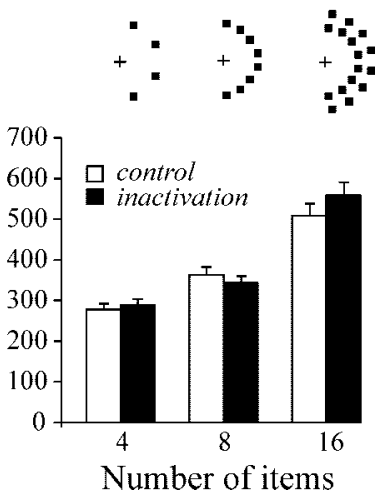

Figure 9. Visual search performance for monkey $\mathrm{M}$ when all items are placed within the same hemifield. Search time for a target defined by a conjunction of color (yellow vs green) and form (diamond vs square) is defined as the interval between the search pattern onset and the start of the saccade landing on the target (bar height is mean search time; error bar indicates SE). Spatial layout of items is represented above each display size for the contralesional $(A)$ and ipsilesional $(B)$ sides. The contralesional field is on the left. White bars correspond to control data; black bars correspond to data after LIP inactivation. Asterisk indicates corrected $p<0.05$.

patients with visual extinction, the contralesional stimulus has to lead the ipsilesional one by $200 \mathrm{msec}$ to be reported as having appeared first, as opposed to $<25 \mathrm{msec}$ in normal subjects. Much more subtle effects have been observed on saccadic choice, but the population of patients tested in this second study no longer showed extinction and had normal temporal order judgments (Ro et al., 2001). A $50 \mathrm{msec}$ target onset asynchrony was sufficient to produce an equal probability of left and right saccades. Thus, despite the differences between the tasks used, the effects of LIP inactivation that we obtained (on the order of $180 \mathrm{msec}$ ) appear closer to those found in patients with extinction than in patients with parietal lesions but no extinction.

The effects of LIP inactivation can also be contrasted with those of FEF lesions. Schiller and Chou (1998) reported a 116 msec target onset asynchrony for balanced choice, suggesting that the FEF is involved in saccade target selection. However, one important difference is that in their study, the proportion of contraversive saccades always approached 100\%, whereas after LIP inactivation contralateral saccade probability remained low, even with target onset asynchronies of $280 \mathrm{msec}$, which are much larger than the monkeys' normal saccade latencies. Furthermore, we found a large proportion of errors on interleaved single contralateral saccade trials. Because such omissions were observed only in this task and not during the memory-guided saccade task, it suggests that after LIP inactivation, the overall task context created a "virtual" competition between the two sides even on single target trials. It is not clear whether the differences in the magnitude of the effects of FEF and LIP lesions are attributable to the fact that Schiller and Chou (1998) made nonreversible lesions and tested the animals in a chronic stage rather than in an acute stage as in the present study, or whether this reveal a genuine difference in the contribution of the two regions to saccade target selection.

\section{Visual search}

Visual search also involves competing stimuli, but in contrast with the above task, irrelevant visual information has to be filtered out 
to localize a single designated target among distractors. In a visual search display, when potential distractors are homogeneous and compete weakly with the designated target, search time is brief and independent of the number of distractors. LIP inactivation has no effect on search time in this so-called "pop-out" or "parallel" visual search (Treisman and Gelade, 1980). However, with many stimuli in the display possessing some attributes of the target (e.g., its color or its shape) and competing strongly with one another, the focus of visual attention and, as in the present study, the eyes will shift from one location to the next until the stimulus with the proper conjunction of attributes is found. Our results reveal an important role of LIP in this serial search process, but the nature of this role needs to be identified more precisely. Clearly, we observed an ipsilesionally biased oculomotor strategy in one of the two monkeys. Whether this bias has motor or attentional origin is open to debate. The differences in search time between the contralesional and ispilesional fields remained present even when the oculomotor bias was factored out (by looking only at trials in which search was initiated toward the hemifield of the target). Furthermore, in the monkey showing the strongest ipsilateral bias, the contralesional impairment remained when the visual competition was confined to a single hemifield. Altogether, these results suggest the implication of an attentional component to the target selection deficit in the contralesional hemifield. Interestingly, in the FEF, where neuronal responses during search tasks have been related to target selection, it has been shown that the visual selection process can be dissociated from saccadic response selection in the activity of visually responsive neurons (Thompson et al., 1996; Murthy et al., 2001). This suggests that LIP may participate in parallel with the FEF in both covert and overt orienting during visual search.

In conclusion, muscimol inactivation of LIP produces deficits in saccadic target selection, consistent with the hypothesis that LIP represents a salience map of potential eye movement targets in the contralateral visual field. These deficits are specific to LIP because inactivation of a neighboring parietal region, VIP, did not produce any effects in the tasks that we investigated. Our results appear in agreement with human brain imaging experiments suggesting that the intraparietal region is activated more strongly during conjunction search than during single-feature search (Corbetta et al., 1995). Also, patients with parietal lobe lesions show deficits in conjunction but not in feature search (Eglin et al., 1989; FriedmanHill et al., 1995); however, these studies tested covert visual search procedure. Further work will be needed to test whether the contribution of LIP to the target selection is dedicated to a specific effector system, e.g., saccade versus reaching, and whether it is also involved in covert attentional processing.

\section{REFERENCES}

Andersen RA, Asanuma C, Essik G, Siegel RM (1990) Cortico-cortical connections of anatomically and physiologically defined subdivisions within the inferior parietal lobule. J Comp Neurol 296:65-113.

Andersen RA, Brotchie PR, Mazzoni P (1992) Evidence for the lateral intraparietal area as the parietal eye field. Curr Opin Neurobiol $2: 840-846$

Barash S, Bracewell RM, Fogassi L, Gnadt JW, Andersen RA (1991a) Saccade-related activity in the lateral intraparietal area. I. Temporal properties: comparison with area 7a. J Neurophysiol 66:1095-1108.

Barash S, Bracewell RM, Fogassi L, Gnadt JW, Andersen RA (1991b) Saccade-related activity in the lateral intraparietal area. II. Spatial properties. J Neurophysiol 66:1109-1124.

Ben Hamed S, Duhamel J-R, Bremmer F, Graf W (2002) Visual receptive field modulation in the lateral intraparietal area during attentive fixation and free gaze. Cereb Cortex 12:234-245.

Bichot NP, Schall JD (1999) Saccade target selection in macaque during feature and conjunction visual search. Vis Neurosci 16:81-89.
Blatt GJ, Andersen RA, Stoner GR (1990) Visual receptive field organization and cortico-cortical connections of the intraparietal area (area LIP) in the macaque. J Comp Neurol 299:421-445.

Bullier J, Schall JD, Morel A (1996) Functional streams in occipitofrontal connections in the monkey. Behav Brain Res 76:89-97.

Colby CL, Duhamel J-R, Goldberg ME (1996) Visual, presaccadic and cognitive activation of single neurons in monkey lateral intraparietal area. J Neurophysiol 76:2841-2842.

Corbetta M, Shulman GL, Miezin FM, Petersen FE (1995) Superior parietal cortex activation during spatial attention shifts and visual feature conjunction. Science 270:802-805.

Dias EC, Segraves MA (1999) Muscimol-induced inactivation of monkey frontal eye field: effects on visually and memory-guided saccades. J Neurophysiol 81:2191-2214.

Di Pellegrino G, De Renzi E (1995) An experimental investigation on the nature of extinction. Neuropsychologia 33:153-170.

Duhamel J-R, Bremmer F, Ben Hamed S, Werner G (1997) Spatial invariance of visual receptive fields in parietal cortex neurons. Nature 389:845-848.

Duhamel J-R, Colby CL, Goldberg ME (1998) Ventral intraparietal area of the macaque: congruent visual and somatic response properties. J Neurophysiol 79:126-136.

Eglin M, Robertson LC, Knight RT (1989) Visual search performance in the neglect syndrome. J Cognit Neurosci 4:372-381.

Felleman DJ, Van Essen DC (1991) Distributed hierarchical processing in the primate cerebral cortex. Cereb Cortex 1:1-47.

Friedman-Hill SR, Robertson LC, Treisman A (1995) Parietal contributions to visual feature binding: evidence from a patient with bilateral lesions. Science 269:853-855

Goldberg ME, Bisley J, Powell KD, Gottlieb J, Kusunoki M (2002) The role of the lateral intraparietal area of the monkey in the generation of saccades and visuospatial attention. Ann NY Acad Sci 956:205-215.

Gottlieb JP, Kusunoki M, Goldberg ME (1998) The representation of visual salience in monkey parietal cortex. Nature 391:481-484

Hays AV, Richmond BJ, Optican LM (1982) A UNIX-based multipleprocess system for real-time data acquisition and control. WESCON Conf Proc 2:1-10.

Judge SJ, Richmond BJ, Chu FC (1980) Implantation of magnetic search coils for measurements of eye position: an improved method. Vision Res 20:535-538.

Karnath H-O (1988) Deficits of attention in acute and recovered visual hemi-neglect. Neuropsychologia 26:27-43.

Kusunoki M, Gottlieb J, Goldberg ME (2000) The lateral intraparietal area as a salience map: the representation of abrupt onset, stimulus motion, and task relevance. Vision Res 40:1459-1468.

Li C-SR, Mazzoni P, Andersen RA (1999) Effect of reversible inactivation of macaque lateral intraparietal area on visual and memory saccades. J Neurophysiol 81:1827-1838.

Lynch JC, McLaren JW (1989) Deficit of visual attention and saccadic eye movements after lesions of parietooccipital cortex in monkey. J Neurophysiol 61:74-90.

Lynch JC, Graybiel AM, Lobeck LJ (1985) The differential projection of two cytoarchitectonic subregions of the inferior parietal lobule of macaque upon the deep layers of the superior colliculus. J Comp Neurol 235:241-254.

Malpeli JG (1999) Reversible inactivation of subcortical sites by drug injection. J Neurosci Methods 86:119-128.

Martin JH, Ghez C (1999) Pharmacological inactivation in the analysis of the central control of movement. J Neurosci Methods 86:145-159.

Mazzoni P, Bracewell RM, Barash S, Andersen RA (1996) Motor intention activity in the macaque's lateral intraparietal area. I. Dissociation of motor plan from sensory memory. J Neurophysiol 75:1233-1241.

Murthy A, Thompson KG, Schall JD (2001) Dynamic dissociation of visual selection from saccade programming in frontal eye field. J Neurophysiol 86:2634-2637.

Ro T, Rorden C, Driver J, Rafal R (2001) Ipsilesional biases in saccades but not perception after lesions of the human inferior parietal lobule. $\mathrm{J}$ Cognit Neurosci 13:920-929.

Rorden C, Mattingley JB, Karnath H-O, Driver J (1997) Visual extinction and prior entry: impaired perception of temporal order with intact motion perception after unilateral parietal damage. Neuropsychologia 35:421-433.

Schiller PH, Chou I (1998) The effects of frontal eye field and dorsomedial frontal cortex lesions on visually guided eye movements. Nat Neurosci 1:248-253.

Snyder LH, Batista AP, Andersen RA (1998) Change in motor plan, without a change in the spatial locus of attention, modulates activity in posterior parietal cortex. J Neurophysiol 79:2814-2819.

Thompson KG, Hanes DP, Bichot NP, Schall JD (1996) Perceptual and motor processing stages identified in the activity of macaque frontal eye field neurons during visual search. J Neurophysiol 76:4040-4055.

Treisman AM, Gelade G (1980) A feature-integration theory of attention. Cognit Psychol 12:97-136. 